Service social

\title{
Compte rendu d'expériences internationales
}

\section{Josiane Deveaud, Anny Gracient et Hyam Samaha-Kahi}

Volume 39, numéro 1, 1990

Le groupe ici et ailleurs

URI : https://id.erudit.org/iderudit/706464ar

DOI : https://doi.org/10.7202/706464ar

Aller au sommaire du numéro

Éditeur(s)

École de service social de l'Université Laval

ISSN

1708-1734 (numérique)

Découvrir la revue

Citer cet article

Deveaud, J., Gracient, A. \& Samaha-Kahi, H. (1990). Compte rendu d'expériences internationales. Service social, 39(1), 168-182. https://doi.org/10.7202/706464ar

\section{Résumé de l'article}

Cet article présente trois expériences de service social des groupes qui ont été menées en France et au Liban.

Josiane Deveaud nous fait d'abord connaître une utilisation innovatrice des TUC (Travaux d'Utilité Collective) dans les îles de Ré, Aix et Oléron. Des actions de groupe conduites conjointement par des travailleurs sociaux, des jeunes, des partenaires sociaux et municipaux, permettent à des jeunes issus de milieux défavorisés, de s'engager dans un processus d'insertion professionnelle et sociale.

Par ailleurs, à Saint-Ouen-L'Aumône, le Service des Retraités, appuyé par le Centre Communal d'Action Sociale, développe le travail social de groupe avec les aides-ménagères pour le maintien à domicile des personnes très âgées. Anny Gracient nous fait part de cette initiative et fait ressortir les effets positifs de cette intervention de groupe, tant au niveau de la qualité du travail des aides-ménagères qu'au plan de leur développement personnel.

Finalement, Hyam Samaha-Kahi montre comment différentes formes de groupes peuvent être génératrices de potentiels humains et de ressources collectives parmi la population libanaise aux prises avec les difficultés et les souffrances provoquées par la guerre. L'auteure témoigne des efforts déployés pour humaniser les conditions de vie dans les centres de regroupement des déplacés. 


\section{Compte rendu d'expériences internationales}

Josiane Deveaud, éducatrice spécialisée, Mission Locale de La Rochelle, France.

\section{"TUC en îles", une expérience de groupe avec des jeunes}

L'expérience présentée ici est menée en France et s'adresse à des jeunes garçons et filles de 16 à 25 ans dans un but d'insertion sociale et professionnelle.

L'entrée des jeunes dans la vie active, après la fin de leur scolarité, est devenue une course d'obstacles, une période d'incertitude et de déstabilisation pour beaucoup d'entre eux. La société doit œuvrer pour que les jeunes ne soient jamais condamnés au chômage, qu'ils aient la possibilité d'acquérir la culture et la formation qui leur permettront de s'adapter aux changements à venir et aient les moyens d'orienter leur vie professionnelle selon leurs goûts et leurs capacités. En 1981, Bertrand Schwartz (1981 : 146) fait le constat qu'une partie importante de ces jeunes est installée dans une marginalité de fait, soit parce qu'ils ne réussissent pas à se faire une place dans la société, soit qu'ils s'excluent eux-mêmes de la vie collective. Ceux qui n'ont pas accès à une insertion active sont renvoyés à une attitude de passivité souvent accentuée par les mesures d'assistance prises en leur faveur.

Ce constat et ces objectifs sont largement partagés par tous ceux qui, dans la société, s'intéressent à la condition actuelle des jeunes et veulent la transformer, et par les jeunes eux-mêmes qui souhaitent que "ça change». Or, ils sont les principales victimes de la crise économique : en France, le taux de chômage est trois fois plus élevé pour les jeunes que pour les adultes. II y a réciproquement une dégradation des chances et des conditions d'insertion qui résultent des mauvaises conditions de leur vie sociale et culturelle. Les adultes 
doivent alors avoir à leur égard une exigence d'écoute et une volonté $d^{\prime}$ action afin de modifier cette situation de " hors jeu économique et social". Les propositions faites par Schwartz (1981) ne visent pas seulement la lutte contre le chômage, mais elles s'intègrent dans un objectif de "remise en jeu économique et sociale »des jeunes. Elles se sont traduites par la mise en place par l'État de certains dispositifs, tels que les Travaux d'Utilité Collective (TUC), instaurés le 16 octobre 1984, en tant qu'action de préformation et de préparation à la vie professionnelle. La création des "TUC » a par exemple aidé les travailleurs sociaux à construire, avec et pour les jeunes, des stratégies diversifiées d'insertion professionnelle et à les associer à la vie de la cité. En établissant un dialogue avec les différents segments du contexte sociétal, en créant les conditions d'un "soutien logistique » local, ils ont pu favoriser un climat de confiance réciproque par et au sein des différents groupes qui se sont constitués, et développer ainsi les énergies et capacités créatrices.

\section{La problématique des jeunes habitant sur le continent}

Le programme "TUC" s'adresse à des jeunes du continent connus de la Mission Locale* et des Services Sociaux de CharenteMaritime. La plupart, sortis de l'école à 16 ans (sans diplôme et sans qualification), en situation d'échec scolaire, connaissaient le chômage et l'insécurité avant même le travail. Ils étaient démobilisés par rapport à un projet professionnel et en conflit avec leurs parents. La majorité d'entre eux étaient issus de milieux sociaux défavorisés depuis des générations : pères chômeurs ou manœuvres, de tradition ouvrière, peu qualifiés ou immigrés; mères souvent dépendantes des circuits d'aide sociale. Les familles avaient souvent des conditions de vie difficiles sur le plan financier et un fonctionnement générateur de séparations ou d'abandons, entraînant carences et insécurité sur le plan affectif.

Ces jeunes étaient confrontés aux images véhiculées par les médias et aux valeurs qui ont cours dans leur environnement. Ils ressentaient violemment les décalages entre les conditions de vie de leur famille et de leur groupe social et celui de l'ensemble de la société. Ils reprenaient souvent à leur propre compte le mépris et la dévalorisation dont sont victimes leurs parents. Pour affirmer leur identité, ils se retrouvaient en bandes souvent "désactivées " et pouvaient avoir des conduites délinquantes. Ils étaient à peu près totalement désorganisés quant à leur temps libre, et avaient souvent des sentiments d'inutilité.

II m'a semblé qu'une proposition de "TUC » en secteur touristique pendant le temps des vacances, pourrait être une réponse 
souple à leur faisceau de problèmes. En échange de travaux, les jeunes pourraient bénéficier de vacances et effectuer des apprentissages sociaux favorisant leur insertion sociale et professionnelle. Le "TUC » serait alors " expérimenté » de manière innovatrice dans une double dimension : professionnelle et sociale.

Dans leur nouveau statut de stagiaire, précaire il est vrai, mais officiel, légitime, négocié et contracté, dans le cadre de la Mission Locale et avec les municipalités, les jeunes pourraient s'engager dans des processus d'apprentissage d'un nouveau rôle : celui de " travailleur ". Ils auraient ainsi l'occasion d'expérimenter des comportements utiles aux collectivités qui les emploient, ainsi qu'à euxmêmes puisqu'ils apprendraient à s'organiser et à gérer leur temps libre à leur propre profit, tout en tenant compte de leurs valeurs personnelles et d'une culture qu'ils veulent construire selon d'autres modèles.

Les objectifs professionnels proposés correspondaient aux besoins des jeunes; ils tenaient compte de leur isolement, marginalité, inactivité, de leurs sentiments d'inutilité et de leurs souhaits d'agir "pour que cela change ". II s'agissait donc de créer des conditions pour qu'ils occupent, même temporairement, un statut de travailleur, et apprennent à organiser autrement la vie au quotidien et leur intégration à l'environnement social. C'est grâce au travail entrepris au sein de leurs groupes pour réussir leurs projets individuels que "les jeunes pourraient apprendre à parler, à participer, à négocier, à s'aider les uns les autres, et à s'aider eux-mêmes en aidant les autres " (Schwartz, 1961). Ils apprendraient aussi à prendre des décisions, à développer une certaine autonomie et à devenir conscients des composantes actuelles de leur vie personnelle et sociale.

Parallèlement, cette action visait à la transformation des pratiques d'accueil des municipalités qui acceptaient d'engager des jeunes pour faire des travaux utiles à la collectivité. Elles étaient prêtes à prendre part par le biais d'un tutorat offert aux jeunes, à un certain type de préformation professionnelle sur le terrain et à travailler avec eux dans une démarche "préventive". Le programme visait aussi à mobiliser les élus et les nouveaux partenaires autour des besoins particuliers des jeunes.

\section{Le groupe de jeunes}

Des jeunes ont été repérés par des travailleurs sociaux et invités à participer, pendant l'été, à des travaux divers sur des îles touristiques de la région : Ré, Oléron et Aix (entretien de campings, sanitaires, plages, voirie, espaces verts). Deux animateurs soutenaient les jeunes dans leur action. Quatorze jeunes ont participé au projet en 1986, 
18 en 1987, 26 en 1988, et 35 en 1989. Des réunions de préparation se sont tenues sur le continent, entre mai et juin, pour faire connaissance et pour que chacun présente ses difficultés. Durant l'été, les réunions de groupe ont été programmées à tous les quinze jours, à l'occasion d'un repas. Elles ont évité l'isolement des jeunes et une rupture trop brutale avec leur milieu habituel de vie. Ces rencontres ont ainsi contribué à leur insertion dans les îles, en plus de faciliter la mise en place de projets concrets et de certaines évaluations.

Dans l'ensemble, les travaux proposés aux jeunes avaient été préalablement négociés avec chaque commune. Les contrats ont été respectés. La " non diversité » du travail effectué a engendré parfois une certaine lassitude. Cependant, tous ont pu acquérir des compétences réelles et faire preuve de leur intégration aux réalités du travail. D'autres tâches plus valorisantes leur ont ensuite été confiées : mise en page de brochures, participation à l'organisation d'expositions, travaux de secrétariat, etc. La nature des relations de travail entre les jeunes et certains employés communaux a été variable, allant de l'entente "parfaite» au mode plus conflictuel. C'est par le travail réalisé dans le groupe que les jeunes ont pu s'aider les uns les autres par leurs critiques ou par leurs encouragements. L'impact a peut-être été plus fort que d'éventuelles " admonestations " ou remarques des employés en situation d'autorité. Est-ce dû au processus " de socialisation par identification à l'autre », présent chez tout jeune qui cherche, pour se faire accepter, à gagner l'approbation du groupe?

Contrairement à leur expérience scolaire, dont ils avaient d'ailleurs été souvent exclus ou qu'ils avaient subie passivement, ces jeunes ont pu, sur les îles, être actifs et en prise directe avec le monde du travail. II leur est devenu possible de s'engager dans un réel processus d'apprentissage. En effectuant un travail, à temps partiel, même peu qualifié mais utile à la collectivité, ils ont pu identifier leurs ressources, leurs manques, et se sont approprié des informations nécessaires pour enrichir et développer leurs connaissances. C'est grâce à l'aide et à la compréhension de ceux qui, dans les communes, les ont encadrés (cantonniers, gérants de camping), qu'ils ont pu dépasser certaines étapes, voire certains blocages, et évaluer ensemble leur progression, leurs acquisitions et la validité de nouveaux comportements.

L'adaptation à la vie sur l'île semble avoir été assez difficile pour les jeunes. Du jour au lendemain, nombre des aspects de leur cadre habituel changèrent. D'une vie organisée autour des copains et des distractions quotidiennes, ils se retrouvaient seuls sur les terrains de camping, contraints de gérer leur budget, compte tenu du coût élevé de la vie sur les îles. Pour la plupart d'entre eux, il s'agissait d'une 
première expérience de travail. L'organisation de leur temps libre les a souvent désappointés. Ils n'étaient pas habitués aux distractions proposées dans ces milieux et l'appréhension de l'inconnu accroissait leur sentiment d'insécurité. Ils ont réussi progressivement à " prendre en charge " leurs loisirs et les ont organisés de façon collective.

Grâce au groupe, les jeunes ont pu s'aider mutuellement à utiliser les ressources locales et à avoir des activités qui correspondaient à leurs besoins et attentes. Ils ont ainsi organisé des sorties en mer (voilier), de pêche, et ont fait de l'équitation, du tennis. Ceci corrobore l'opinion de William Schwartz (1961) selon laquelle "dans la relation d'aide c'est le client qui seul possède les moyens réels d'atteindre ses propres fins ».

\section{Les résultats}

La plupart des jeunes ont mené leur «TUC » à terme. Plusieurs ont négocié des travaux plus valorisants avec les Mairies, d'autres ont eu la possibilité de prolonger leur "TUC » dans la commune qui les avait accueillis. Les abandons ont pour la plupart été causés par la " fragilité " de certains jeunes ou par un manque de relations avec le responsable du «TUC » sur la commune. Il a donc été souhaité qu'un " tuteur " par commune soit nommé pour faciliter les relations des jeunes avec leur employeur.

Les communes, sensibilisées aux difficultés particulières de ces jeunes ayant parfois tendance à la marginalisation, ont manifesté une volonté réelle de les aider dans leur démarche d'insertion professionnelle. II a été décidé d'élargir les Travaux d'Utilité Collective à la participation des jeunes aux aspects culturels de leur environnement immédiat, par exemple leur collaboration à des manifestations culturelles locales, pour acquérir des connaissances dans les domaines de l'histoire, du théâtre, etc. Cette étape était jugée indispensable pour accéder à un niveau général, nécessaire pour leur insertion dans le milieu social environnant.

L'action s'élargissant aux trois îles, le maintien et l'extension d'un travail de groupe permanent entre les différents partenaires ont été perçus comme une nécessité. Après chaque été, plusieurs jeunes ont maintenu les contacts avec la Mission Locale pour entreprendre des démarches liées à leur formation professionnelle. Certains d'entre eux ont pu effectuer des stages de qualification dans des secteurs divers comme le bâtiment, I'horticulture ou les emplois de collectivité. D'autres se sont engagés dans la recherche d'un emploi, et certains sont même entrés dans le monde du travail assez rapidement. 


\begin{abstract}
Note
* Les Missions Locales sont appelées à développer leur activité au bénéfice de l'ensemble des jeunes de 16 ans et plus, qui ne sont ni scolarisés ni en apprentissage, ni titulaires d'un emploi permanent. Elles ont vocation à se préoccuper de l'ensemble des problèmes d'insertion sociale qui se posent aux jeunes: vie quoditienne, logement, santé, etc. Dotées de moyens spécifiques, pouvant disposer de personnels, les Missions Locales sont constituées, à l'initiative des collectivités locales, de préférence sous la forme d'une Association, avec accord des Pouvoirs Publics qui participent à leur financement en collaboration avec les Collectivités Locales.
\end{abstract}

\title{
Références
}

SCHWARTZ, Bertrand (1981). L'insertion professionnelle et sociale des jeunes: rapport au Premier ministre. Paris : La documentation française.

SCHWARTZ, William (1961). "The Social Worker in the Group » : 146-177, dans The Social Welfare Forum. New York : Columbia University Press.

Anny Gracient, directrice du Centre Communal d'Action Sociale, SaintOuen-I'Aumône, France.

\section{Travail social de groupe et maintien à domicile des personnes âgées}

Le projet de groupe présenté prend place à Saint-Ouen-l'Aumône, ville de 18500 habitants, située à $30 \mathrm{~km}$ au nord de Paris. Il avait pour but de mettre à la disposition de la population âgée un personnel spécialisé pour l'aider à demeurer à domicile et à faire face aux problèmes liés à la vie quotidienne, tant sur le plan matériel, comme les travaux domestiques, que sur les plans affectif, de soutien psychologique et $d^{\prime}$ 'isolement. Vingt nouvelles aides-ménagères ont alors été recrutées par le Centre Communal d'action sociale (CCAS) ${ }^{1}$.

La complexité de la relation et le travail quotidien auprès de personnes très âgées, souvent en grandes difficultés physiques ou mentales, place fréquemment les aides-ménagères elles-mêmes en situation de détresse, entraînant de nombreux arrêts pour cause de maladie. L'objectif du projet devenait alors de donner une nouvelle dimension au travail d'aide-ménagère, c'est-à-dire dépassant la 
pratique traditionnellement axée sur les tâches ménagères pour s'orienter vers une action plus professionnelle au sens gérontologique.

Un groupe d'aides-ménagères a été mis en place par un travailleur social formé à la pratique professionnelle du service social des groupes pour amener ces femmes à s'engager elles-mêmes dans une dynamique de professionnalisation. Un entretien individuel avec chacune d'elles les a préparées à comprendre ce qui serait en jeu dans leur participation aux réunions de groupe. Ces entretiens ont fait ressortir les principaux problèmes qu'elles doivent affronter : I'isolement face à la relation avec les personnes âgées, leur peur de trouver des personnes décédées, leur difficulté à répondre à certaines exigences des personnes âgées quant aux travaux ménagers d'un autre temps, tels que le nettoyage hebdomadaire derrière les gros meubles, le retournement quotidien de lourds matelas de laine, le lavage à la main des draps chez les personnes énurétiques, celles-ci ne voulant pluss investir dans l'achat d'une lessiveuse.

Des réunions mensuelles, d'une durée d'une heure trente, ont été organisées, suivies régulièrement par quinze personnes. Plusieurs ' d'entre elles ont pris des rôles actifs dans ce groupe, en donnant des conseils pour un ménage plus rationnel, en expliquant la demande affective des personnes âgées, puis en sachant prendre des distances par rapport à l'organisation du temps de travail de façon à pouvoir accompagner la personne âgée pour les courses plutôt que de les faire à leur place, par exemple.

Ces rôles leur ont donné un certain prestige qui a rejailli sur le groupe, c'est-à-dire que le regard de la "Mairie " s'est transformé. Les comportements individuels de certaines aides-ménagères se sont modifiés: une jeune femme au bord de la marginalité, étant difficilement acceptée par les personnes âgées, est maintenant réclamée. Après un an de travail, une aide-ménagère a décidé de participer à une journée de formation sur l'accompagnement des mourants, journée proposée par le service et organisée par l'Association de Gérontologie de Paris $13^{\mathrm{c}}$. Pour lever sa peur de se retrouver seule et de faire face à une situation nouvelle dans un lieu inconnu, nous avons décidé de l'accompagner à cette journée; médecins, infirmières et aides-ménagères de toute la France en composaient l'auditoire. L'aide-ménagère a constaté qu'elle comprenait les interventions. Elle a pris conscience que sa connaissance concrète du terrain rejoignait la théorie énoncée. Au cours de la réunion suivante, le compte rendu de cette journée, fait par l'aide-ménagère au groupe, a convaincu d'autres aides-ménagères de l'intérêt d'aller à ce genre d'activités de formation. Plusieurs d'entre elles ont alors décidé de s'engager dans des stages de trois jours. À leur retour, elles ont 
travaillé les contenus de ces formations avec le travailleur social du service. Au cours des réunions suivantes, elles ont pu rapporter leurs nouvelles connaissances à l'ensemble du groupe. Elles sont devenues, en quelque sorte, des formatrices à l'intérieur du groupe. Lors d'un jeu de rôle, elles ont appris les gestes adaptés pour lever une personne âgée alitée. $D$ 'autres ont pu dénouer des situations difficiles en entamant, avec la participation du service, une levée de tutelle abusivement décidée par la famille.

Chacune des aides-ménagères est engagée dans un processus d'apprentissage "professionnel " si nécessaire pour fournir une prestation de qualité. Le risque d'isolement des aides-ménagères est réduit par le travail du groupe.

\section{Évaluation}

Une première évaluation a montré une réelle transformation du comportement professionnel des aides-ménagères. La satisfaction évoquée par les bénéficiaires du maintien à domicile en est aussi le reflet. On peut souligner que des personnes âgées ont demandé l'intervention du service malgré qu'elles devaient payer elles-mêmes les frais maximum exigés, en raison de leurs ressources plus élevées. La présence du service a permis à un plus grand nombre de personnes âgées de mourir à leur domicile plutôt qu'à l'hôpital. Ces personnes ont été " accompagnées " jusqu'à leur fin par les familles, les aidesménagères et le service.

La constitution de petits groupes a permis de créer un lieu de travail et d'expression pour les aides-ménagères, ce qui les a aidées à faire face à l'angoisse de l'accompagnement jusqu'aux derniers instants. Les aides-ménagères ont pu aussi contribuer à rassurer et à déculpabiliser les familles. Le travail social de groupe a développé la confiance des aides-ménagères entre elles et envers le service. Elles ont acquis des possibilités d'expressions verbales et des compétences techniques. Elles sont détentrices de connaissances et d'informations qu'elles peuvent partager et mettre à la disposition du service pour évaluer, avec d'autres partenaires, les besoins des personnes âgées.

La transformation de leur comportement vers une compétence accrue du rôle d'aide-ménagère, par l'apprentissage réciproque et grâce à l'aide mutuelle, les a conduites à prendre conscience des valeurs de chacune liées à leur responsabilité dans cette fonction, et à modifier les valeurs du groupe.

Le degré d'absentéisme des aides-ménagères a diminué. Des liens de solidarité se sont créés entre elles. La Mairie les considère comme appartenant à une structure, à un corps dont elle reconnaît la 
dimension professionnelle. L'équipe peut maintenant développer un programme plus étendu de maintien à domicile.

\begin{abstract}
Note
1 Le CCAS est un établissement public dont le rôle est d'animer une action générale de prévention et de développement social dans la commune, en liaison étroite avec les institutions publiques et privées. Cet organisme relève de la commune et le maire en est président de droit.
\end{abstract}

Hyam Samaha-Kahi, directrice et professeure, École libanaise de formation sociale, Université SaintJoseph, Beyrouth, Liban.

\title{
Service social des groupes et humanisation des conditions de vie dans les centres de regroupement des déplacés
}

Avoir une signification dans le monde est l'aspiration la plus profonde de l'âme humaine, plus profonde que la faim et la soif physiques, et quand un homme l'a perdue, il n'est plus un homme.

Alan Paton

Le but du présent article est de montrer comment la vie d'un groupe peut être génératrice de potentiels humains et de ressources collectives.

Les hostilités de la guerre au Liban ont entraîné, au cours des quinze dernières années, des déplacements massifs de populations. On retrouve une collectivité aigrie par des souffrances qui dépassent toute imagination, des familles désemparées et des milliers d'handicapés, de "sans travail », de "sans logement ». Le service social se trouve alors confronté à une nouvelle réalité qui dépasse de beaucoup les possibilités et les ressources existantes; le travailleur social est ainsi appelé à user de toute son ingéniosité pour mobiliser I'ensemble des ressources et des potentialités existantes dans un milieu en difficulté, en vue de l'aider à y faire face. 
L'expérience que nous décrivons ici a été pensée et menée par un groupe d'enseignants de l'École libanaise de formation sociale (ELFS) auprès de 83 familles déplacées (373 personnes) qui vivent dans cinq centres de regroupement différents. Nous appelons "centre de regroupement " tout lieu de rassemblement de populations déplacées (immeubles détruits ou abandonnés; centres à usage public ou communautaire : école, administration publique, couvent, etc.). La majorité des personnes qui habitent ces centres ont été obligées de quitter en catastrophe leurs lieux habituels de résidence, suite à des actes d'hostilité exercés envers elles. D'un jour à l'autre, elles se sont retrouvées sans abri, sans travail, sans biens, ayant tout perdu, quelquefois même des membres de leur famille. Exilées à l'intérieur de leur propre pays, ces personnes ont, pour la plupart, trouvé refuge dans des régions plus sécuritaires, où elles ont été logées dans des locaux de fortune, soi-disant pour quelques jours. Cependant, la majorité d'entre elles occupent toujours ces locaux, étant incapables d'acquérir une autre résidence et également incapables de retourner à leur région d'origine à cause de la situation de guerre qui y prévaut.

\section{Quelques caractéristiques de la population prise en charge}

Les populations qui vivent dans les centres dont nous discutons proviennent du Liban-Sud (région du Chouf et région Est de Saïda). Le nombre moyen d'enfants par famille est de quatre. La majorité de la population est essentiellement jeune ( $34 \%$ a moins de 20 ans). Par ailleurs, $10 \%$ de la population a plus de 60 ans. L'espace vital ${ }^{1}$ dont dispose une famille ne dépasse pas en moyenne 16 mètres carrés. On y mange, dort, cuisine, reçoit, prend son bain; aucune intimité n'est possible. De plus, les conditions de logement sont souvent insalubres.

\section{L'action engagée}

La première étape de notre action consistait à apprivoiser cette population aigrie par l'ampleur des difficultés vécues. Nous avons procédé par observation participante, cherchant à faire exprimer, par les personnes directement concernées, leurs sentiments et besoins. Cette période, qui a duré trois mois, nous a aussi permis de connaître les lieux, d'identifier les problèmes vécus et d'en évaluer l'ampleur, puis d'élaborer des perspectives d'action.

Plusieurs niveaux de problèmes ont été identifiés, certains liés aux conditions matérielles de vie (conditions objectives), d'autres aux aspects psychosociaux et relationnels (vécu ou conditions subjec- 
tives). Parmi les problèmes d'ordre matériel, on retrouve ceux touchant les aspects socio-économiques: perte de biens, épuisement de petites économies, chômage forcé, décès des membres productifs, handicap permanent, dévaluation de la livre libanaise et inflation. D'autres sont reliés aux conditions de logement : inconfort, exiguïté, partage d'un nombre réduit de sanitaires, espace vital limité. Des problèmes de santé sont aussi causés ou aggravés par la guerre : handicaps physiques, mutilations, maladies psychosomatiques, maladies de peau, manque de nutrition, etc. D'autres touchent l'éducation : retard scolaire, arrêt des études. Certains problèmes sont enfin liés aux aspects psychosociaux et relationnels; ils se manifestent surtout à travers les perturbations de rôles à l'intérieur de la famille, les difficultés d'adaptation, les sentiments d'isolement, d'infériorité, de honte, de frustration, de déracinement, de marginalisation. On retrouve aussi un fort sentiment d'insécurité dû à la peur d'être chassé à tout moment de locaux sur lesquels les occupants ne détiennent aucun droit.

Nous avons aussi procédé à une investigation exhaustive de toutes les structures et organisations sociales existantes dans les localités avoisinantes à chaque centre (services offerts, conditions, personnes responsables, etc.). Ceci nous a permis de constater que ces structures offraient souvent les mêmes services, étaient pour la plupart peu ou mal connues par les habitants des centres et manquaient de coordination au niveau de l'organisation et de la distribution des services.

Pour améliorer la situation stressante vécue par ces personnes qu'on a arrachées à un environnement avec lequel elles étaient en harmonie, et qu'on a placées dans un nouveau milieu où cette harmonie n'a pas encore été retrouvée, nous avons choisi d'œuvrer à deux niveaux : auprès des personnes, pour les aider à accroître leur propre estime d'elles-mêmes et leurs capacités de faire face aux difficultés et problèmes de la situation présente, puis auprès des réseaux sociaux et des structures organisationnelles, pour les promouvoir et les rendre plus adaptés, plus humains. Nous voulions en quelque sorte travailler dans une perspective globale et intégrée touchant à la fois le développement de la personne et de son environnement.

\section{Objectifs, moyens et techniques}

À court et à moyen terme, nous visions à aider les populations déplacées à améliorer leur cadre de vie (environnement physique), à faire le deuil du.passé, à répondre aux urgences sans tomber dans I'assistance tout court. À plus long terme, nous voulions aider les 
habitants des centres à se procurer un logement individuel, à vivre dans des conditions plus décentes et à s'insérer dans le nouveau milieu d'accueil.

Les moyens utilisés ont été assez diversifiés : intervention individuelle, familiale, de groupe et auprès des réseaux et des structures organisationnelles. Nous avons eu le plus souvent recours à divers types d'interventions de groupe. La philosophie et les concepts théoriques qui sous-tendent cette méthode s'adaptent parfaitement à notre réalité orientale; ils s'inscrivent dans la ligne des habitudes et des valeurs qui existent déjà dans nos sociétés et qu'il $s^{\prime}$ agit de sauvegarder, positiviser et renforcer.

Parmi les techniques auxquelles nous avons fait appel et qui nous ont aidés dans la réalisation de certaines tâches, mentionnons les techniques du support social. Nous avons œuvré tout au long de notre expérience à renforcer les différents liens de support social qui existent déjà et à en trouver de nouveaux car nous sommes persuadés, comme plusieurs auteurs, que le support social peut amortir le stress. Nous avons travaillé avec plusieurs réseaux dont le réseau naturel de soutien (famille élargie, voisinage, amis). Ce réseau, très important dans la société libanaise, a permis de résorber de nombreux problèmes pendant les quinze années de guerre; on a beaucoup parlé du "miracle » libanais, c'en est un aspect. Nous avons aussi collaboré avec le système de soutien dans la communauté (associations, parti, paroisses). Enfin, nous avons très souvent fait appel aux groupes d'entraide (regroupement de pairs vivant des situations semblables) et avons constaté que ces groupes sont d'une grande efficacité.

Différents autres types de groupes ont aussi été mis en place. Par exemple, un comité local a été formé dans chaque centre de regroupement. Composé de représentants des habitants du centre, il a pour tâche de s'occuper des travaux d'entretien et d'assurer l'implication directe des résidents dans les travaux d'aménagement que nous avons engagés pour améliorer les conditionnements des logements (réparation des locaux, installation de canalisations d'eau, d'électricité, badigeonnage, installation de sanitaires, ouverture d'égoûts). Ce comité représente aussi le centre auprès des organismes d'aide; il peut négocier, revendiquer ou refuser une aide quelconque selon les priorités.

Nous avons également organisé, dans deux centres, plusieurs groupes de "rattrapage scolaire " destinés aux enfants qui présentaient un retard. Pour nous aider dans cette activité, nous avons fait appel aux étudiants plus âgés qui habitent le centre et à quelques jeunes du voisinage immédiat. Cette tâche assurée en commun a permis d'amorcer une relation amicale avec le milieu d'accueil. 
Dans une des localités qui abrite trois des centres, nous avons participé à la création d'un "Comité de coordination » regroupant tous les organismes d'aide existants en vue d'assurer une meilleure coordination et une plus grande adéquation des services.

Nous voulions aussi aider les familles à faire l'acquisition de maisons individuelles; il fallait alors trouver des alliés influents et perspicaces. Nous avons réussi à former un "Comité d'appui " composé de représentants des centres, de prêtres responsables des paroisses qui accueillent les centres en même temps que responsables d'un organisme d'aide, de nous-mêmes comme partenaires, d'un avocat habitant l'un des centres et qui agit à titre de personneressource pour tous les problèmes administratifs. D'autres personnes-ressources sont invitées selon les sujets à l'ordre du jour. Ce Comité a réussi à mobiliser une instance religieuse importante dans le pays et influente dans ce domaine. Plusieurs démarches ont été entreprises pour étudier de près la faisabilité d'un projet qui aiderait ces familles à s'approprier des logements (coopération, banque).

Un groupe de jeunes filles a été formé dans l'un des centres, dans le but de suivre des cours de couture industrielle qui leur assurent une formation professionnelle minimale. Dans un autre centre, nous avons motivé un petit groupe de pères de famille à bénéficier d'un projet de réhabilitation économique qui consiste à les aider à louer un terrain, à le cultiver et à en exploiter la production.

\section{Principaux rôles des intervenants sociaux}

Auprès de la population, il s'agissait surtout, pour l'intervenant social, d'agir comme personne-ressource, mobilisateur, facilitateur, médiateur, avocat et organisateur; de toujours partager avec le groupe les responsabilités telles que la détermination des objectifs, la réalisation des activités et leur réajustement en cours de route. II fallait veiller constamment à assurer une réelle décentralisation du pouvoir.

Auprès des organisations et structures sociales, les rôles joués étaient essentiellement les suivants : clarification des réalités, mobilisation des réseaux et potentialités, médiation, recherche d'alliés dans chaque organisme, coordination au niveau de la base, identification des besoins.

Dans tout cela, la qualité de la relation reste plus importante que les techniques elles-mêmes. L'écoute, l'empathie et la congruence demeurent des éléments indispensables à toute relation d'aide. 


\section{Limites et interrogations}

Au cours de cette expérience, nous avons eu à répondre à plusieurs interrogations. Déjà au début de notre action, nous étions face à deux choix qui en conditionnent différemment l'orientation :

- fallait-il aider ces personnes déplacées à s'insérer dans leur nouveau milieu d'accueil ?

— fallait-il aller dans le sens de leur désir spontané, c'est-à-dire les aider à s'organiser de façon provisoire dans l'espoir d'un retour prochain au milieu d'origine, tout en sachant que ce retour dépend de plusieurs considérations politiques et sécuritaires?

À l'époque, nous avions opté pour la première perspective, celle de les aider à s'insérer dans le milieu d'accueil, même si le retour dans leur lieu d'origine est prochain, pour la simple raison qu'une personne a toujours besoin d'implanter ses racines quelque part pour pouvoir fonctionner et croître. II s'agit donc de favoriser le développement d'une capacité d'implantation dans différents milieux. L'avenir doit cesser d'être uniquement fonction d'un sol qui retranche la personne de son passé. L'avenir a besoin d'un projet de devenir pour appartenir à l'Homme.

Cependant, l'insertion nécessite forcément des changements à opérer au double plan du milieu d'accueil et de l'individu ou du groupe accueilli. Ces changements affectent à la fois le modèle d'organisation de la société et les modes d'adaptation de la personne. L'insertion suppose donc autant d'efforts de normalisation que $\mathrm{d}^{\prime}$ intégration. Bien que profondément conscients des limites de notre action face à la complexité du projet, nous n'avons pas hésité un seul instant à choisir la voie difficile, car elle est la seule qui soit humanisante dans le vrai sens du terme.

Dans le monde actuel et au Liban plus spécialement, nous sommes face à un défi, celui de l'exercice de la tolérance et de la démocratie. Cela n'est possible que si nous pouvons recréer des communautés humaines où prévalent les deux valeurs fondamentales de tout travail social : le droit et la responsabilité.

Le service social doit avoir le courage de proposer des alternatives et de générer à chaque instant, et face à chaque situation, de nouveaux moyens susceptibles de promouvoir des pratiques toujours plus humanisantes, une solidarité effective des gens et une recherche continue du bien commun.

Les droits dans une société, sont fonction des efforts déployés en leur faveur, car en définitive, il n'existe de droits que dans la lutte qu'on engage pour les définir, les maintenir et les développer. 
Aujourd'hui, le monde, et le Liban en particulier, n'a plus besoin de décrets ni de textes, mais bien d'une action criante et efficace dans le sens des Droits de l'Homme. II en est de même pour le service social qui a cru pouvoir exister à force de décrets; ce dont il a besoin, c'est d'une action militante de tous les jours.

\section{Note}

\footnotetext{
${ }^{1}$ Nous appelons espace vital la superficie totale dont dispose la famille, à savoir la pièce où loge cette famille, de même que les couloirs, la salle de bain et la cuisine, qui sont en commun.
} 